\title{
ANALYSIS OF PERFORMANCE OF THE NEW TYPE OF FOUR EQUAL OF THE INJECTION ONLINE PRESSURE SEALING FIXTURE
}

\author{
Li Hao rong ${ }^{1}$, Chen Qing ${ }^{*}$, Hu Yi wei ${ }^{1}$, Chen Yu fei ${ }^{2}$ \\ ${ }_{1}^{1}$ Power Engineering and Engineering Thermophysics, Jilin Institute of Chemical technology, Chengde Street, Jilin City, Jilin, China. \\ ${ }^{2}$ Fine Chemicals Plant, Petro China Jilin Petrochemical Corporation, Chengde Street, Jilin City, Jilin, China. \\ *Corresponding Author Email:chenqing0787@126.com
}

This is an open access article distributed under the Creative Commons Attribution License, which permits unrestricted use, distribution, and reproduction in any medium, provided the original work is properly cited

\section{ARTICLE DETAILS}

Article History:

Received 26 June 2018

Accepted 2 july 2018

Available online 1 August 2018

\begin{abstract}
Taking the leakage of steel pipe welding neck flanges with raised face as the research object, the structura characteristics of the traditional fixture and new type of four equal fixture were investigated, and the mechanical properties of the traditional fixture and the new type of four equal fixture were analyzed by using the finite element software Abaqus. The results show that, in the case of the same internal uniform pressure applied to the two kinds of flanges, the maximum circumferential tensile stress and the maximum tensile stress of the bolt of the new type of four equal fixture is $42.2 \%$ and $42.9 \%$ for the traditional fixture, respectively. The new type of four equal fixture is superior to the traditional fixture in both pressure bearing capacity and stress concentration. Therefore, it is of great significance to investigate the mechanical properties and concept design of the new type of four equal fixture for the wide application of pressure sealing technology.
\end{abstract}

KEYWORDS

Online leak sealing, traditional fixture, four equal fixture, finite element analysis

\section{INTRODUCTION}

Petrochemical and other industries at domestic and foreign to run the production process, pipeline leakage has always been an unavoidable problem. At present, the widely used technology for solving pipeline leakage in China is injection-type pressure sealing technology [1,2]. The injection-type pressure sealing technology originated in the United States. In 1920, American Klein Furman developed a leak sealing method under pressure in a pipeline at Newport's shipyard and expanded it to other industries [3-5]. China's pressure sealing technology began in the 1950s. It was the use to pressure welding to treat leaking pressure equipment in the steel industry [6].

However, it is forbidden for a long time in China's chemical industry, it obtain developed and applied of the injection-type pressure seal until the early 1980s [7]. And during the national "6th Five-Year Plan" period, it completed the development of model-to-technical innovation of pressure sealing technology, and was subsequently included in one of the 19861990 national key 70 new technology promotion projects $[8,9]$. It has been reported that in China, the technology has successfully eliminated more than 500,000 leakages of various types, and economic losses have been recovered totalling more than 60 billion yuan, resulting in huge social benefits [10-12]. In 2007, China announced the world's first national industry standard HG/T20201-2007 "Pressure Sealing Technical Specifications", at an international in which the new theory of fixture design is an advanced level [13].

In this paper, the mechanical properties of the traditional fixture and the new type of four equal fixture under different pressures were analyzed by using the finite element software Abaqus. It is of great significance to the investigate results for the Promotion and application of the Injection Online Pressure Sealing technology.

\section{MODEL AND MATERIAL PARAMETERS}

In this paper, taking the leakage of steel pipe welding neck flanges with raised face as the research object, and medium pressure saturated steam is taken. The operating pressure is $3.0 \mathrm{MPa}, 5.0 \mathrm{MPa}$, and $8.0 \mathrm{MPa}$, respectively. Traditional fixtures, new type of four equal fixtures and flange material parameters are shown in Table1.

Table 1: Material performance parameters

\begin{tabular}{|lcll|}
\hline element & $\begin{array}{l}\text { Traditional } \\
\text { fixture }\end{array}$ & Four equal fixture & Flange \\
\hline $\begin{array}{l}\text { Material type } \\
\begin{array}{l}\text { Modulus of } \\
\text { elasticity/MPa }\end{array}\end{array}$ & $2.1 \times 10^{5}$ & Q235-B & Q235-C \\
$\begin{array}{l}\text { Poisson's ratio } \\
\text { Diameter/mm }\end{array}$ & 0.3 & 0.3 & $2.13 \times 10^{5}$ \\
$\begin{array}{l}\text { Temperature } /{ }^{\circ} \mathrm{C} \\
\text { Operating }\end{array}$ & 220 & 105 & 0.3 \\
pressure $/ \mathrm{MPa}$ & $3 、 5 、 8$ & $3 、 5 、 8$ & 105 \\
\hline
\end{tabular}

\section{FINITE ELEMENT MODEL ESTABLISHMENT}

This article uses abaqus software to establish a finite element analysis model. Because the traditional pressure sealing fixture is a two-part structure composed of two semicircles, select the $1 / 2$ model that establishes of the overall structure of the traditional fixture as the research object, model symmetry plane set symmetrical constraints, apply fixed restraints to the lug plates on both sides $[14,15]$. The setting parameters are as follows: Unit type is C3D8R, Poisson's ratio 0.3, Elastic modulus $2.1 \times 105 \mathrm{MPa}$, Apply uniformly distributed pressure to the inner wall of the fixture $\mathrm{P}=3.0 \mathrm{MPa}, \mathrm{P}=5.0 \mathrm{MPa}$ and $\mathrm{P}=8.0 \mathrm{MPa}$, finite element model of the $1 / 2$ traditional fixture shown in Figure 1. 


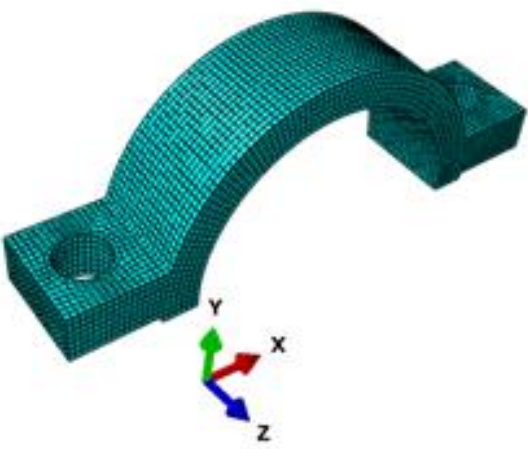

Figure 1: Finite element model of the $1 / 2$ traditional fixture

The new type of four equal fixture structure is mainly composed of three parts: fixture, sealing film and fastening bolt. It is mainly applied to various damage seals of oil, water and other liquid media [16]. Because the new type of four equal pressure sealing fixture is a four-part structure composed of four quarter-arc structures, select the $1 / 4$ model that establishes of the overall structure of the new type of four equal fixture as the research object, apply fixed restraints to the lug plates on both sides. Set the parameters as above, finite element model of the $1 / 4$ new type of four equal fixture shown in Figure 2.

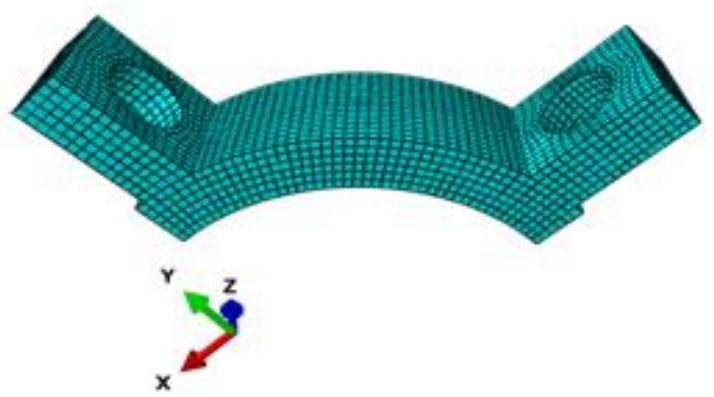

Figure 2: Finite element model of the 1/4 new type of four equal fixture

\section{RESULTS OF COMPARATIVE ANALYSIS}

The circumferential tensile stress of the traditional fixture under different presses is shown in Figure 3. The arc portion of the fixture is subjected to most of the uniform pressure, and the circumferential tensile stress of the fixture increases first and then decreases, and the middle position of the arc part of the fixture is subject to the greatest circumferential tensile stress. As can be seen from the peak of the three curves, the circumferential tensile stress of the fixture increases with the increase of the pressure. When the pressure $\mathrm{P}=8.0 \mathrm{MPa}$, the circumferential tensile stress value of the fixture is the largest, the maximum value is $46.73 \mathrm{MPa}$. The bolt tensile stress of the traditional fixture under different pressure is shown in Figure 4.

The bolts are subjected to a large tensile stress, from overall view, the tensile stress of the bolt changes of a parabolic shape, increasing first to a maximum value and decreasing to a minimum value, and the more obvious the curve changes as the pressure increases, the steeper the shape of the curve and the greater the peak value. It can be seen that the tensile stress of the bolt increases as the pressure increases and the local stress concentration near the maximum, the greater the pressure and the more obvious the stress concentration. resulting in stress concentration. When the pressure $\mathrm{P}=8.0 \mathrm{MPa}$, the tensile stress value of the bolt is the largest, the maximum value is $167.2 \mathrm{MPa}$.

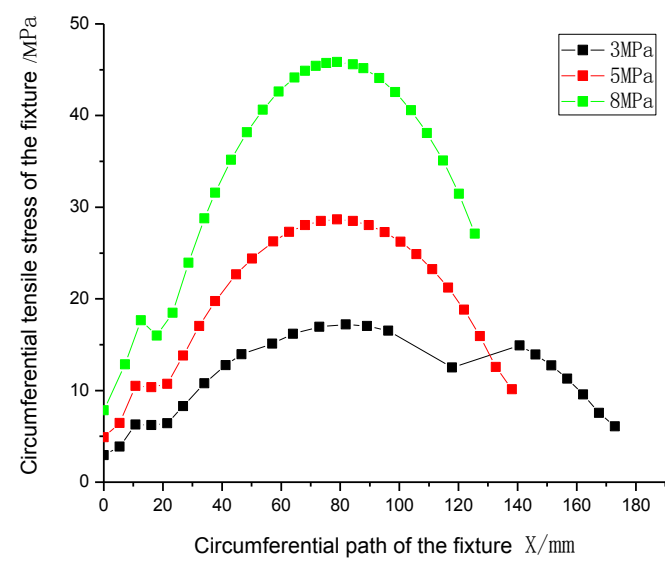

Figure 3: Circumferential Tensile stress of the traditional fixture under different pressures

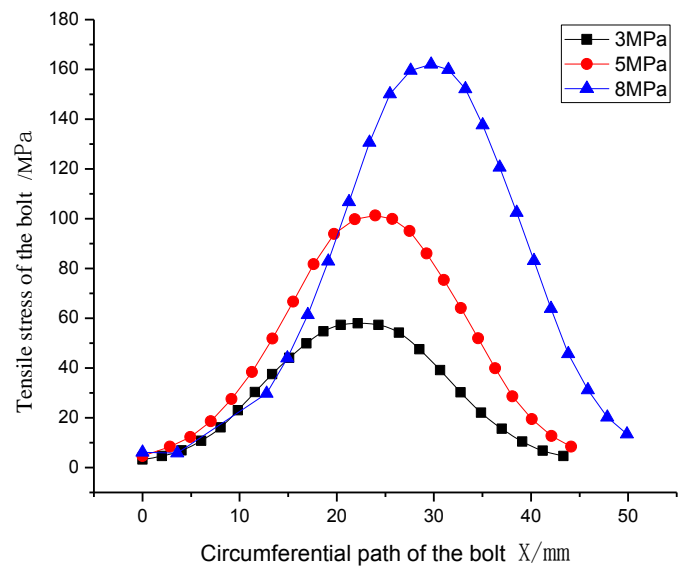

Figure 4: Bolt tensile stress of the traditional fixture under different pressures

The circumferential tensile stress of the new type of four equal fixture under different presses is shown in Figure 5. The circumferential tensile stress of the new type of four equal fixture is symmetrically distributed from the middle to the two sides, and the circumferential tensile stress value of the fixture is the largest in the middle. It can be seen from the trend of the graph that the circumferential tensile stress value of the fixture increases first and then decreases and increases to the maximum value. The circumferential tensile stress value of the fixture increases with the increase of the pressure. When the pressure $\mathrm{P}=8.0 \mathrm{MPa}$, the circumferential tensile stress value of the fixture is the largest, the maximum value is $19.71 \mathrm{MPa}$.

Tensile stress of bolt of the new type of four equal fixture under different pressure is shown in Figure 6. The tensile stress of the bolt suffered gradually increase according to the parabolic trend, when the maximum value is reached, it tends to stabilize in a small interval, and then shows a downward trend. As the pressure increases, the peak of the curve also increases. When the pressure $\mathrm{P}=8.0 \mathrm{MPa}$, the tensile stress value of the bolt is the largest, the maximum value is $71.73 \mathrm{MPa}$. Comparison of analysis results of mechanical properties of fixtures under different pressures are shown in Table 2 


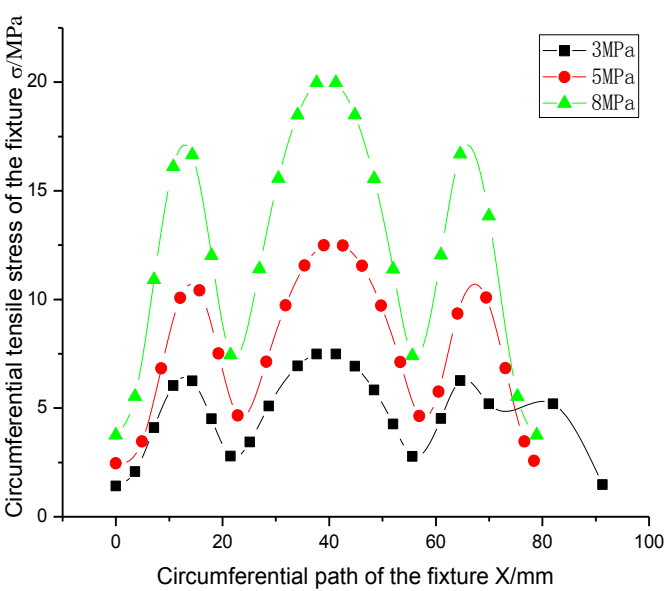

Figure 5: Circumferential tensile stress of the new type of four equal fixture under different pressures

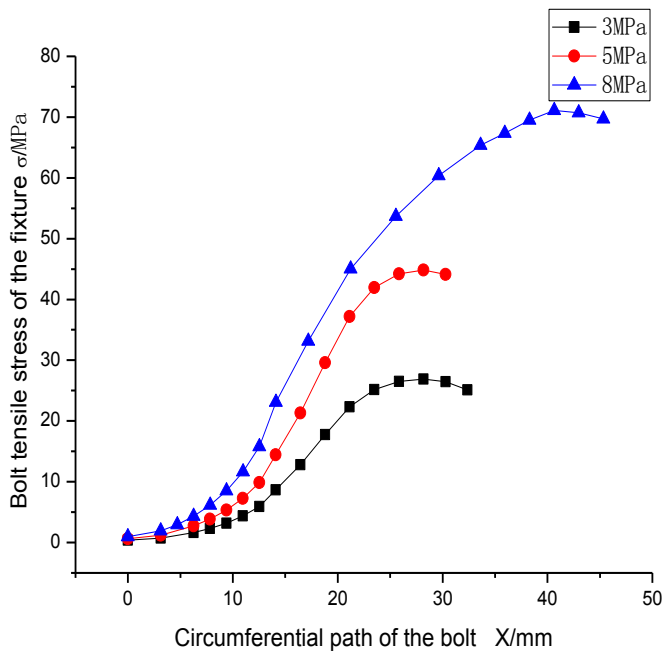

Figure 6: Tensile stress of bolt of the new type of four equal fixture under different pressures

Table 2: Comparison of analysis results of mechanical properties of fixtures under different pressures

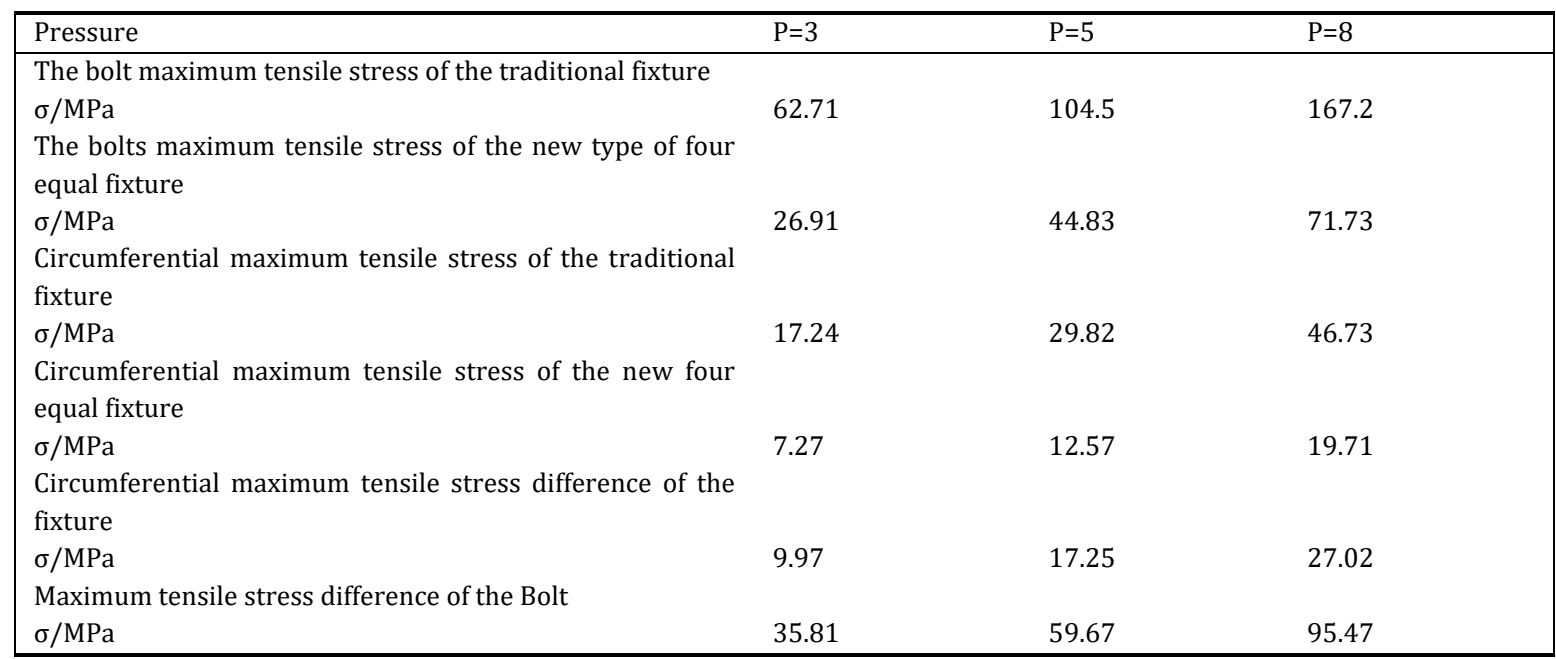

\section{CONCLUSION}

Through the above analysis and Table 2 , it can be clearly seen that the new type of four equal fixture is superior to the traditional fixture in both pressure bearing capacity and stress concentration. In the structural design, the new type of four equal fixture can also better solve the shortcomings such as poor surface adhesion, loose fastening bolts, etc., and increase the sealing performance and seal life of the fixture. Under the condition that the same internal uniform pressure is applied to the traditional fixture and the new type of four equal fixture, The analysis shows that within the allowable range of allowable stress of bolt, the new type of four equal fixture can withstand higher pressure and increase the pressure bearing capacity of the fixture. The maximum circumferential tensile stress and the maximum tensile stress of the bolt of the new type of four equal fixture is $42.2 \%$ and $42.9 \%$ for the traditional fixture, respectively.

\section{REFERENCES}

[1] Zhao, H.M., Hu, Y.W. 2010. Research on sealed-up method of pressure leakage accident emergency and terminology translation. China Safety Science and Technology, 6 (1), 154-157.

[2] Hu, Y.W. 2006. Introduction to pressure seal engineering. Journal of Rolling and Sealing, (02), 79-82.

[3] Otegui, J.L., Cisilino, A., Rivas, A.E., Chapetti, M., Soula, G. 2002. Influence of multiple sleeve repairs on the structural integrity of gas pipelines. International Journal of Pressure Vessels \& Piping, 79 (11), 759 765.
[4] Dai, Y.F. 1985. On-line plugging technology of furmanite company. Petrochemical Equipment, (05), 26-32.

[5] Elaoud, S., Hadj-Taïeb, L., Hadj-Taïeb, E. 2010. Leak detection of hydrogen-natural gas mixtures in pipes using the characteristics method of specified time intervals. Journal of Loss Prevention in the Process Industries, 23 (5), 637-645.

[6] Zhao, Z.H. 2011. Status quo and development trend of pressure seal technology equipment for chemical enterprises. Modern Chemical Industry, $31(02), 9-12$.

[7] Hu, Y.W. 2005. Study on theoretical calculation formula of pressure seal gland. Chemical Machinery, 32 (01), 18-21.

[8] Li, X., Yang, M., Hu, Y.W. 2010. Research on object-oriented design method of pressurized seal fixture. Journal of Rolling and Sealing, 35 (08), 88-90.

[9] Hu, Y.W. 1998. Dynamic sealing technology. Beijing: National Defense Industry Press, 593-595.

[10] Yu, F.Y. 2015. Research on application of natural gas pipeline pressure plastic injection fixtures. Master thesis of Northeast Petroleum University.

[11] Hu, Y.W. 2006. Introduction to pressure seal engineering. Jilin Institute of Chemical Technology, 23 (01), 39-43.

[12] Shaikh, M.M., AlSuhaimi, A.O., Hanafiah, M.M., Ashraf, M.A., Fantoukh, A., AlHarbi, E. 2017. Leachable Volatile Organic Compounds from Polyethylene Plumbing Plastic Pipes: a case study of Medina Al Munawarah, Saudi Arabia. Acta Chemica Malaysia, 1 (1), 01-03. 
[13] HG/T20201-2007 Pressure seal technical specifications. Beijing: China Plan Press.

[14] Ma, X., Zheng, J.Y. 2004. A new method for the design of injection-type sealing fixture. Chemical Engineering \& Machinery, 31 (02), 100-103.

[15] De'nan, F., Nazri, F.M., Hashim, N.S. 2017. Finite Element Analysis on Lateral Torsional Buckling Behavior Oi I-Beam with Web Opening. Engineering Heritage Journal, 1 (2), 19-22.

[16] Liu, F.L., Cai, X.J. 2011. Design and analysis of a new sealing fixture with pressure sealing. New Technology and New Technology, (11), 55-59.

\section{ABOUT THE AUTHORS}

First Author:Li Haorong (1992-), male, master candidate of jilin institute of chemical technology, main research direction is injection on-line pressure sealing technology. E-mail: LHR814186316@163.com

Second Author: Chen Qing (1964-), male, professor of jilin institute of chemical technology, main research direction is process equipment fluid seal technology. E-mail: chenqing0787@126.com

Third Author: Hu Yiwei (1956-), male, professor of jilin institute of chemical technology, pressure seal technology expert, main research direction is injection on-line pressure sealing technology. E-mail: huyiwei@sina.com 